\title{
THE DISTRIBUTION OF GENOTYPES PRODUCED BY ALLELES SEGREGATING AT A NUMBER OF LOCI
}

\author{
PETER O'DONALD* \\ Nuffield Unit of Medical Genetics, University of Liverpool, P.O. Box 147, Liverpool L69 $3 B X$
}

Received 14.v.70

\section{INTRODUCTION}

IT is sometimes implied that the segregation of alleles at a number of loci affecting a metrical character should produce a normal distribution. For example, Falconer (1960, p. 109) assumes that any metrical character will have a normal distribution if it is scaled appropriately. Often, of course, there is a transformation to turn a particular distribution into a normal one. But this will not necessarily produce a scale appropriate to the study of biometrical genetics. Most of the theoretical work on biometrical genetics has assumed that a gene has an additive effect on a character. And on the simplest assumptions the distribution produced by $n$ loci segregating for two alleles with additive effects will not be even approximately normal. For example, suppose the gene frequency is $p$ for all $n$ loci, each with an average effect $\alpha$. The third moment about the mean is

$$
\mu_{3}=2 n \alpha^{3} p q(q-p)
$$

and it is only zero (which it must be if the distribution is normal) if $p=1$. The fourth moment is

$$
\mu_{4}=2 n \alpha^{4} p q[1+6(n-1) p q] .
$$

For a normal distribution $\mu_{4}=3 V^{2}$, where $V$ is the variance. In this case $\mu_{4}$ becomes approximately equal to $3 V^{2}$ only when $n$ is very large. If, therefore, we scale a distribution to make it approximately normal, then necessarily we should have made the assumption of additive genetic effects invalid, for genotypic distributions are never normal when the genes are additive.

There are three necessary parameters in the genetic model: the gene frequency, the average effect and the number of loci. They will determine the deviations from normality. Conversely, the deviations from normality may be used to estimate the parameters. Mather (1949) and Cooke and Mather (1962) showed that it was possible to estimate the "number of effective factors ", $n$, by the variances of crosses between inbred lines if the genes have the same effects at all effectively distinct loci. Robson (1956) showed that the fourth cumulant can also be used to estimate $n$ from these crosses. Making the further assumption that the gene frequencies are alike at all loci, the same calculation can be done for the distribution of genotypes in a population mating at random. The second, third and fourth cumulants of a binomial distibution are

$$
\begin{aligned}
& K_{2}=2 n p q \alpha^{2} \\
& K_{3}=2 n p q(p-p) \alpha^{3} \\
& K_{4}=2 n p q(1-6 p q) \alpha^{4}
\end{aligned}
$$

* Present address: Department of Genetics, Milton Rd., Cambridge CB4 1XH. 
and equating the cumulants to the $k$ statistics of an observed distribution we get

$$
k_{3}^{2} / k_{4} k_{2}=\frac{1-4 p q}{1-6 p q}
$$

so that we estimate the frequency of heterozygotes by

and the number of loci by

$$
2 \hat{p q}=\frac{k_{3}^{2}-k_{4} k_{2}}{3 k_{3}^{2}-2 k_{4} k_{2}}
$$

$$
\hat{n}=k_{2}^{3} /\left(k_{2}^{2}-k_{4} k_{2}\right)
$$

Obviously some fairly drastic assumptions have been made to obtain these estimates. Apart from assuming that the average effects and gene frequencies are the same at all loci, we have also assumed that the distribution of genotypes is known. It is my object in this paper to discuss the errors that are likely to be introduced by these assumptions, their likely magnitudes and ways of dealing with them.

\section{VARIATION IN AVERAGE EFFEGTS}

If the values of $\alpha$ vary between the different loci, we may write for the $i$ th locus $\alpha_{i}=\alpha\left(1+x_{i}\right)$ where the values of $x_{i}$ are distributed around a mean of zero. Assuming that the gene frequencies are constant, then we have

$$
\begin{aligned}
& K_{2}=2 p q \alpha^{2} \sum_{i=1}^{n}\left(1+x_{i}\right)^{2} \\
& K_{2}=2 n p q \alpha^{2}\left(1+V_{x}\right) \\
& K_{3}=2 n p q \alpha^{3}\left(1+3 V_{x}+\mu_{3}\right) \\
& K_{4}=2 n p q(1-6 p q) \alpha^{4}\left(1+6 V_{x}+4 \mu_{3} x+\mu_{4} x\right) \text {. } \\
& \text { If } \mu_{3} x=0 \text { and } \mu_{4} x=3 V_{x}^{2} \text {, then } \\
& K_{2}=2 n p q \alpha^{2}\left(1+V_{x}\right) \\
& K_{3}=2 n p q(q-p) \alpha^{3}\left(1+3 V_{x}\right) \\
& K_{4}=2 n p q(1-6 p q) \alpha^{4}\left(1+6 V_{x}+3 V_{x}^{2}\right) .
\end{aligned}
$$

The estimate of the number of loci is therefore

$$
\frac{2 n p q\left(1+V_{x}\right)^{3}}{(1-4 p q)\left(1+3 V_{x}\right)^{2}-(1-6 p q)\left(1+V_{x}\right)\left(1+6 V_{x}+3 V_{x}^{2}\right)} \text {. }
$$

If $V_{x}$ is fairly small, so that $V_{x}^{2}$ is negligible, then the estimate of $n$ becomes

which equals

$$
\frac{2 n p q\left(1+3 V_{x}\right)}{2 p q\left(1+9 V_{x}\right)-V_{x}}
$$

or roughly

$$
n\left\{\frac{\left(1+3 V_{x}\right)}{1+V_{x}(9-1 / 2 p q)}\right\}
$$

$$
n\left(1+3 V_{x}\right)
$$


Thus if $\hat{n}=k_{2}^{3} /\left(k_{3}^{2}-k_{4} k_{2}\right)$ then $n=\hat{n} /\left(1+3 V_{x}\right)$ approximately. The effect of variation between loci in the additive effects of the alleles is therefore to give an estimate of $n$ which is too large and should be reduced by a factor of about $1 /\left(1+3 V_{x}\right)$.

\section{VARIATION IN GENE FREQUENGIES}

Since the gene frequencies must vary between loci, the genotypic frequencies of a diploid organism are given by

$$
\prod_{i=1}^{n}\left(p_{i}+q_{i}\right)^{2}
$$

The cumulant generating function is

$$
2 \sum_{i=1}^{n} \log _{e}\left(q_{i}+p_{i} e^{\theta}\right)
$$

and the cumulants are then found to be

$$
\begin{aligned}
& \kappa_{2}=2 \sum_{i} p_{i} q_{i} \alpha_{i}^{2} \\
& \kappa_{3}=2 \sum_{i} p_{i} q_{i}\left(q_{i}-p_{i}\right) \alpha_{i}^{3} \\
& \kappa_{4}=2 \sum_{i} p_{i} q_{i}\left(1-6 p_{i} q_{i}\right) \alpha_{i}^{4} .
\end{aligned}
$$

If the values of $\alpha_{i}$ are all constant, the cumulants can be evaluated in terms of the mean gene frequency and the moments about the mean of the gene frequency distribution. Putting

$$
\begin{aligned}
\sum_{i} p_{i} & =n \bar{p} \\
\sum_{i}\left(p_{i}-\bar{p}\right)^{2} & =n \sigma_{p}^{2} \\
\sum_{i}\left(p_{i}-\bar{p}\right)^{3} & =n \mu_{3} p \\
\sum_{i}\left(p_{i}-\bar{p}\right)^{4} & =n \mu_{4} p
\end{aligned}
$$

we obtain

$$
\begin{aligned}
& \kappa_{2}=2 n \bar{p} \bar{q}-2 n \sigma_{p}^{2} \\
& \kappa_{3}=2 n \bar{p} \bar{q}(\bar{q}-\bar{p})-6 n \sigma_{p}^{2}(\bar{q}-\bar{p})+4 n \mu_{3 p} \\
& \kappa_{4}=2 n \bar{p} \bar{q}(1-6 \bar{p} \bar{q})-2 n \sigma_{p}(7-36 \bar{p} q)+24 n \mu_{3 p}(\bar{q}-\bar{p})-12 n \mu_{4 p} .
\end{aligned}
$$

These are quite general expressions which, multiplied by the average effects, $\alpha$, give the cumulants of the genotypic distribution.

The problem is now to give values to $\sigma_{p}^{2}, \mu_{3 p}$ and $\mu_{4 p}$. We must therefore choose an appropriate distribution for the gene frequencies. Suppose 
for example that $p_{i}$ is rectangularly distributed in the range 0 to 1 . Then we have

$$
\begin{aligned}
\bar{p} & =\int_{0}^{1} p d p=\frac{1}{2} \\
\sigma_{p}^{2} & =\int_{0}^{1} p^{2} d p-\bar{p}^{2}=\frac{1}{12} \\
\mu_{3 p} & =0
\end{aligned}
$$

and

$$
\mu_{4 p}=\int_{0}^{1} p^{4} d p-4 \bar{p} \int_{0}^{1} p^{3} d p+6 \bar{p}^{2} \int_{0}^{1} p^{2} d p-3 \bar{p}^{4}=\frac{1}{80} .
$$

The cumulants are therefore

$$
\begin{aligned}
& K_{2}=\frac{1}{3} n \alpha^{2} \\
& K_{3}=0 \\
& K_{4}=-\frac{1}{15} n \alpha^{4}
\end{aligned}
$$

If $k_{3}=0$, then the estimate of $n$ becomes $\hat{n}=-k_{2}^{2} / k_{4}=\frac{5}{3} n$ so that

$$
n=\frac{3}{5} \hat{n}
$$

and the estimate of $n$ as given by the simple formula is therefore too large by the factor $\frac{3}{5}$.

This approach can be made general if the gene frequencies have a beta distribution. If for example

$$
y=\frac{x^{l-1}(1-x)^{m-1}}{B(l, m)}
$$

then provided $l$ and $m$ are greater than one, the frequency density $y$ is zero at the termini of 0 and 1 and has a mode at $(l-1) /(l+m-2)$. The $r$ th moment about zero is given by

The mean is therefore

$$
\mu_{r}^{\prime}=\frac{l(l+1) \ldots(l+r-1)}{(l+m)(l+m+1) \ldots(l+m+r-1)} .
$$

$$
\bar{p} \quad=\frac{l}{l+m} .
$$

If $m$ is made some multiple of $l$, say $m=g l$, then $\bar{p}=1 /(1+g)$. The higher moments about the mean can then be found in terms of $l$ and $g$. It can be shown that

$$
\begin{aligned}
\sigma_{p}^{2} & =\frac{g}{(1+g)^{2}[l(1+g)+1]} \\
\mu_{3 p} & =\frac{2 g(g-1)}{(1+g)^{3}[l(1+g)+1][l(1+g)+2]} \\
\mu_{4 p} & =\frac{3 l g^{2}(1+g)+6 g[g(g-1)+1]}{(1+g)^{4}[l(1+g)+1][l(1+g)+2][l(1+g)+3]} .
\end{aligned}
$$


Since $\bar{p}=\frac{1}{1+g}$ and $\bar{q}=\frac{g}{1+g}$, the expressions for $\sigma_{p}^{2}, \mu_{3 p}$ and $\mu_{4 p}$ can be found in terms of $\bar{p}, \bar{q}$ and $l$. These expressions can then be inserted into the general expressions for the cumulants of the genotypic distribution. After a lot of heavy algebra we get

$$
\begin{aligned}
& K_{2}=\frac{2 \bar{p} \bar{q} l n}{l+\bar{p}} \\
& K_{3}=\frac{2 \bar{p} \bar{q}(\bar{q}-\bar{p}) l^{2} n}{(l+\bar{p})(l+2 \bar{p})} \\
& K_{4}=\frac{2 \bar{p} \bar{q}(1-6 \bar{p} \bar{q}) l^{3} n-2 \bar{p}^{2} \bar{q} l^{2} n}{(l+\tilde{p})(l+2 \bar{p})(l+3 \bar{p})} .
\end{aligned}
$$

As $l \rightarrow \infty$ the gene frequency distribution becomes narrower and narrower and $K_{2} \rightarrow 2 \bar{p} \bar{q} n, K_{3} \rightarrow 2 \bar{p} \bar{q}(\bar{q}-\bar{p}) n$ and $K_{4} \rightarrow 2 \bar{p} \bar{q}(1-6 \bar{p} \bar{q}) n$.

As an example, suppose $\bar{p}=\bar{q}=\frac{1}{2}$. Then

$$
\begin{aligned}
& K_{2}=\ln /(2 l+1) \\
& K_{3}=0 \\
& K_{4}=-\frac{l^{2} n}{(2 l+1)(2 l+3)} .
\end{aligned}
$$

If $l=1$, this gives the results previously obtained for a simple rectangular distribution of gene frequencies. I presume that this is likely to be the most extreme case. Generally I expect $l$ to be greater than one. If therefore we estimate

the true number of loci is

$$
\begin{aligned}
\hat{n} & =-k_{2}^{2} / k_{4} \\
& =\frac{(2 l+3)}{(2 l+1)} n
\end{aligned}
$$

$$
n=\frac{(2 l+1)}{(2 l+3)} \hat{n} .
$$

So for $l=1, n=\frac{3}{5} \hat{n}$, for $l=2, n=\frac{5}{7} \hat{n}$ and for $l=3, n=\frac{7}{9} \hat{n}$. The larger values of $l$ do not lead to estimates of $n$ that are seriously misleading.

The most satisfactory way to proceed would be to determine from observed gene frequencies an empirical distribution and estimate the value of $l$. The general expressions for the cumulants could then be equated to the observed $k$ statistics and the equations solved for $n, \bar{p}$ and $\alpha$ by one of the standard procedures of numerical analysis using a computer. But this would probably be an unnecessarily refined approach to the problem, for the estimate of the number of loci given by this method is never more than of the order of magnitude of the true number. And it may be no less satisfactory to calculate an estimate

$$
\hat{n}=k_{2}^{3} /\left(k_{3}^{2}-k_{4} k_{2}\right)
$$

and then get an idea whether it may be too great or too small as a value for $n$. 
From the general expressions for the cumulants we find that

$$
n=\left\{\frac{(l+\bar{p})(\bar{q} l+\bar{p})(l+1)}{\bar{q}(l+2 \bar{p})^{2}(l+3 \bar{p})}\right\} \hat{n} .
$$

The factor within the brackets is a " correction factor" by which $\hat{n}$ should be multiplied to give the true value of $n$. Table 1 shows the values of the

TABLE 1

\begin{tabular}{|c|c|c|c|c|}
\hline \multirow[b]{2}{*}{$p$} & \multicolumn{4}{|c|}{ Values of $l$} \\
\hline & 1 & 2 & 3 & 4 \\
\hline 0 & $2 \cdot 0000$ & 1.5000 & 1.3333 & $1 \cdot 2500$ \\
\hline $0 \cdot 1$ & $1 \cdot 3058$ & $1 \cdot 1948$ & $1 \cdot 1416$ & $1 \cdot 1111$ \\
\hline $0 \cdot 2$ & 0.9566 & 0.9916 & 0.9996 & $1 \cdot 0022$ \\
\hline 0.3 & 0.7636 & 0.8548 & 0.8954 & 0.9183 \\
\hline $0 \cdot 4$ & 0.6547 & 0.7653 & 0.8222 & 0.8569 \\
\hline 0.5 & $0 \cdot 6000$ & 0.7143 & 0.7778 & 0.8182 \\
\hline 0.6 & 0.5903 & 0.7016 & 0.7653 & 0.8066 \\
\hline $0 \cdot 7$ & 0.6347 & 0.7406 & 0.7994 & 0.8367 \\
\hline 0.8 & 0.7832 & 0.8838 & 0.9312 & 0.9566 \\
\hline 0.9 & $1 \cdot 3100$ & $1 \cdot 4101$ & $1 \cdot 4254$ & $1 \cdot 4131$ \\
\hline
\end{tabular}

correction factor for different values of $\bar{p}$ and $l$. It shows that if the mean gene frequency is between 0.1 and 0.2 or 0.8 and 0.9 then $\hat{n}$ is a reasonably good estimate of $n$ whatever the distribution of gene frequencies. But if the mean gene frequency is between 0.3 and 0.7 then $\hat{n}$ will be too high, and if the mean gene frequency is near to zero or one, it will be too low.

For example, the following statistics were obtained for the distribution of sternopleural bristles in 564 male Drosophila melanogaster (O'Donald, unpublished):

$$
\begin{aligned}
& k_{2}=4 \cdot 591726 \\
& k_{3}=5.049888 \\
& k_{4}=4 \cdot 659676 .
\end{aligned}
$$

The distribution is of course far from being normal according to these figures. We get $2 \hat{p q}=0 \cdot 12178$, so that $\hat{p}=0.6513$. If $l=1$, a mean gene frequency of 0.06513 gives a correction factor for $\hat{n}$ of $1 \cdot 492$. If $l=2$, the corresponding correction factor is $1 \cdot 287$. The uncorrected estimate of $n$ is

$$
\hat{n}=23.58
$$

Hence the corrected estimates of $n$ are

(i) if $l=1$ for the distribution of gene frequencies, then

and (ii) if $l=2$ then

$$
n=35
$$

$$
n=30 \text {. }
$$

Variation in the average effects would, of course, tend to reduce these estimates. If the variance in the average effects were, say, $V_{x}=0 \cdot 1$, then we should have to reduce the estimate of $n$ to about 23 again. Variation in gene frequencies and average effects may therefore tend to cancel each other out to leave $\hat{n}$ as providing an idea of at least the order of magnitude of $n$. 


\section{The Genotypic Distribution}

The calculations of section 3 were carried out using the statistics of a phenotypic distribution of bristles of Drosophila. But unless the heritability (which was unknown in this case) is near to unity, the distribution of phenotypes will not reflect the distribution of genotypes. It is necessary to know the additive genetic variance and also the third and fourth moments as determined by the additive genetic variation alone. The second, third and fourth $k$ statistics can then be found using the formulae given by Fisher (1954, p. 72).

Since the heritability is the regression of breeding values on phenotypic values (Falconer, 1960, p. 166), it might be thought that the second, third and fourth moments of the breeding values (Falconer's term for the additive genetic values) would be easy to calculate. But this is not so. If $A$ is the additive genetic deviation - the "breeding value" - and $P$ the phenotypic deviation, then the regression equation is

$$
E(A / P)=h^{2} P
$$

and the moments of $E(A / P)$ are not those of $A$.

But suppose that $P=A+R$, where $A$ is the additive genetic deviation and $R$ a remainder consisting of dominance and environmental deviations. If so, then $A$ and $R$ will be independent and $E(A)=E(R)=0$. The mean value of the offspring of a parent (whose value is $P$ ) is equal to $\frac{1}{2} A$, as shown by Falconer (1960). Therefore, we get the well known formula

$$
\begin{aligned}
\operatorname{cov}(\bar{o}, p) & =E\left(\frac{1}{2} A P\right) \\
& =\frac{1}{2} E\left(A^{2}\right)+\frac{1}{2} E(A R) \\
& =\frac{1}{2} V_{A}
\end{aligned}
$$

showing that the covariance of the mean of the offspring with a parent is equal to half the additive genetic variance. The higher bivariate moments can be used to give the higher moments of the additive genetic values. Thus

and similarly

$$
\begin{aligned}
\operatorname{cov}\left(\bar{o}^{2}, p\right) & =\frac{1}{4} E\left\{A^{2}(A+R)\right\} \\
& =\frac{1}{4} \mu_{3 A} \\
\operatorname{cov}\left(\bar{o}, p^{2}\right) & =\frac{1}{2} E\left\{A(A+R)^{2}\right\} \\
& =\frac{1}{2} \mu_{3} A
\end{aligned}
$$

$$
\begin{aligned}
\operatorname{cov}\left(\bar{o}^{3}, p\right) & =\frac{1}{8} \mu_{4 A} \\
\operatorname{cov}\left(\bar{o}^{2}, p^{2}\right) & =\frac{1}{4} \mu_{4 A}+\frac{1}{4} V_{A} V_{R} \\
\operatorname{cov}\left(\bar{o}, p^{3}\right) & =\frac{1}{2} \mu_{4 A}+\frac{3}{2} V_{A} V_{R} .
\end{aligned}
$$

Thus we have two estimates for both $\mu_{3 A}$ and $\mu_{4 A}$ :

and

$$
\begin{aligned}
\mu_{3 A} & =4 \operatorname{cov}\left(\bar{o}^{2}, p\right) \\
& =2 \operatorname{cov}\left(\bar{o}, p^{2}\right)
\end{aligned}
$$

$$
\begin{aligned}
\mu_{4 A} & =8 \operatorname{cov}\left(\bar{o}^{3}, p\right) \\
& =6 \operatorname{cov}\left(\bar{o}^{2}, p^{2}\right)-\operatorname{cov}\left(\bar{o}, p^{3}\right) .
\end{aligned}
$$


They can be calculated from the same data as those used to calculate $h^{2}$ and $V_{A}$.

If values of $R$ are normally distributed, the higher moments and cumulants of the additive genetic values are easy to calculate. Since $A$ and $R$ are assumed to be independent, any cumulant of $P$ is simply the sum of the two corresponding cumulants of $A$ and $R$. Thus

$$
\begin{aligned}
& K_{2 P}=K_{2 A}+K_{2 R} \\
& K_{3 P}=K_{3 A}+K_{3 R} \\
& K_{4} P=K_{4 A}+K_{4 R}
\end{aligned}
$$

and if $R$ is normally distributed, then $K_{3 R}=K_{4 R}=0$, so that

$$
\begin{aligned}
& K_{2 A}=h^{2} K_{2} P \\
& K_{3 A}=K_{3 P} \\
& K_{4 A}=K_{4} P .
\end{aligned}
$$

Thus if the environmental factors cause variation which is normally distributed, the second cumulant of the genotypic values is increased but the higher cumulants are unchanged.

Suppose, therefore, in the example of the bristle numbers, that $h^{2}=0 \cdot 8$. We should then have the following $k$ statistics of the additive genetic values:

giving

$$
\begin{aligned}
& k_{2 A}=3 \cdot 673381 \\
& k_{3 A}=5.049888 \\
& k_{4 A}=4 \cdot 659676
\end{aligned}
$$

$$
\hat{n}=5 \cdot 9 \simeq 6 \text { loci }
$$

or about one-quarter of the estimate if $h^{2}=1$. Unfortunately there are no data to calculate $h^{2}$ for bristles in this population of Drosophila melanogaster.

\section{Summary}

1. The second, third and fourth cumulants of the genotypic distribution of a quantitative character can be used to estimate the number of loci which control the variation in the character.

2. Variations between the loci in the average effects of the alleles will produce estimates of the number of loci which are too large.

3. Variations in the gene frequencies can be allowed for in the calculations if it is assumed that the gene frequencies at the different loci follow a $\beta$ distribution.

4. The cumulants of the distribution of additive genetic effects can be calculated from data on parents and offspring, or they can be calculated from the cumulants of the phenotypic distribution if it is assumed that environmental factors give rise to normally distributed variations. 


\section{REFERENGES}

COOKE, P., AND MATHER, K. 1962. Estimating the components of continuous variation.

II. Genetical. Heredity, 17, 211-236.

FALCONER, D. s. 1960. Introduction to Quantitative Genetics. Oliver and Boyd, Edinburgh and London.

FISHER, R. A. 1954. Statistical Methods for Research Workers, 12th edition. Oliver and Boyd, Edinburgh and London.

Mather, K. 1949. Biometrical Genetics. Methuen \& Co., London.

ROBSON, D. S. 1956. Applications of the $k_{4}$ statistics to genetic variance component analyses. Biometrics, 12, 433-444. 